\title{
Towards Early Detection of Depression through Smartphone Sensing
}

\author{
Kennedy Opoku Asare \\ kennedy.opokuasare@oulu.fi \\ University of Oulu \\ Oulu, Oulu
}

\author{
Aku Visuri \\ University of Oulu \\ Oulu, Oulu \\ aku.visuri@oulu.fi
}

\author{
Denzil S.T. Ferreira \\ University of Oulu \\ Oulu, Oulu \\ denzil.ferreira@oulu.fi
}

\begin{abstract}
Major depressive disorder is a complex and common mental health disorder that is heterogeneous and varies between individuals. Predictive measures have previously been used to predict depression in individuals. Given the complexity, heterogeneity of major depressive disorder in individuals, and the scarcity of labelled objective depressive behavioural data, predictive measures have shown limited applicability in detecting the early onset of depression. We present a developed system that collects similar smartphone sensor data like in previous predictive analysis studies. We discuss that anomaly detection and entropy analysis methods are best suited for developing new metrics for the early detection of the onset and progression of major depressive disorder.
\end{abstract}

\section{CCS CONCEPTS}

- Human-centered computing $\rightarrow$ Ubiquitous and mobile computing; - Applied computing $\rightarrow$ Life and medical sciences.

\section{KEYWORDS}

Mobile Sensing, Mental Health, Depression

\section{ACM Reference Format:}

Kennedy Opoku Asare, Aku Visuri, and Denzil S.T. Ferreira. 2019. Towards Early Detection of Depression through Smartphone Sensing. In Adjunct Proceedings of the 2019 ACM International foint Conference on Pervasive and Ubiquitous Computing and the 2019 International Symposium on Wearable Computers (UbiComp/ISWC '19 Adjunct), September 9-13, 2019, London, United Kingdom. ACM, New York, NY, USA, 4 pages. https://doi.org/10.1145/3341162.3347075

Permission to make digital or hard copies of all or part of this work for personal or classroom use is granted without fee provided that copies are not made or distributed for profit or commercial advantage and that copies bear this notice and the full citation on the first page. Copyrights for components of this work owned by others than ACM must be honored. Abstracting with credit is permitted. To copy otherwise, or republish, to post on servers or to redistribute to lists, requires prior specific permission and/or a fee. Request permissions from permissions@acm.org.

UbiComp/ISWC '19 Adjunct, September 9-13, 2019, London, United Kingdom (C) 2019 Association for Computing Machinery.

ACM ISBN 978-1-4503-6869-8/19/09 ..\$15.00

https://doi.org/10.1145/3341162.3347075

\section{INTRODUCTION}

Major Depressive Disorder(MDD) is one of the most common mental health disorder, globally affecting about 300 million people [16]. MDD is known to increase the risk of poor studies and job performance [14], the risk of suicide in extreme cases, and also worsens the outcome of conditions such as Parkinson's disease [12] and Heart diseases [17], leading to a negative impact in the well-being of individuals.

Notwithstanding the increased public awareness and the availability of effective pharmacological and cognitive behaviour therapies, MDD affecting many individuals goes undetected and untreated. The ability to detect the early onset of MDD in individuals will have significant impact on addressing MDD. The current advancement in smartphone sensing, Experience Sampling Method (ESM) and human behaviour modelling using affective computing, multi-modal data fusion and machine learning algorithms $[5,18,19]$ has enabled the instrumentation smartphones to unobtrusively detect MDD in individuals beyond laboratory confinements.

Previous studies $[5,6,18,19]$ have used Predictive Analysis (PA) and statistical analysis to study the association between digital biomarkers and MDD. However, the applicability of these methods to early detection of MDD is limited due to the scarcity of objective depressive behavioural data from affected individuals, thus the PA results in low predictive and statistical power. Moreover, PA predicts the future with an assumption that the future will be as it was in the past, which is unrealistic[4] in complex challenges like MDD, whose symptoms is highly heterogeneous and varies between individuals[8]. In addition, these previous studies have relied on assessments of depression such as the Patient Health Questionnaire (PHQ) [15], and the Beck Depression Inventory (BDI) [1]. These depression assessments are subjective, have not changed in the last 30 years [19], and were created largely by clinical consensus and not based on empirical evidence[9]. Even with these limitations, these methods remain the gold standard for assessing the progress of MDD in individuals.

We present preliminary work towards the development of a system for early detection of the onset of depression. We collect similar data; smartphone sensor data, PHQ-9, BDI and BIG-5[13] as previous studies [5, 6, 18, 19], however, we 
take a different approach in analysing the data using entropy analysis $[2,3,10]$, and anomaly detection[11] to develop new methods and metrics that are insightful in understanding the complex vectors of MDD for early detection of the onset and progression of MDD.

\section{RELATED WORK}

Several studies have carried out investigations of the statistical significance and correlation of digital biomarkers in detecting and predicting metal health disorders. Systematic literature reviews $[5,18]$ have shown that digital biomarkers presented in Table 1 have shown promising results on the detecting mental health diseases.

In a 9-week study[19] that enrolled 43 female and 40 male college undergraduates $(N=83$, mean age $=20.13$, std $=2.31)$, participants' digital biomarkers including sleep duration, physical activity, phone unlock duration, stationary time, conversation time, GPS coordinates, were collected using a smartphone application. As a ground truth for depression, the smartphone application administered self reported PHQ8 , and PHQ-4 questionnaires at defined intervals. The study used regression analysis and ANOVA to conclude among others, that students with higher scores of PHQ-8 are more likely to use their phones at study places $(r=0.391, p<0.001)$ when compared to all day phone usage $(r=0.282, p=0.010)$, have irregular sleep patterns, and visits fewer places.

A similar study[6] conducted with 79 participants (58 females, and 21 males, aged 18-25) collected physical activity, and GPS coordinates and self reported PHQ-9 scale using smartphone application. Features comprising Location variance, Time spent moving, total distance, average moving speed were computed from the GPS coordinates data. In addition, the GPS locations where clustered using DBSCAN clustering analysis, and number of unique locations and location entropy of each unique location cluster were extracted. A combination of PHQ-9 scores and the GPS data features as predictors, showed significantly higher predictive power in predicting depression with Support Vector Machine (SVM) classifier than using PHQ-9 scores alone or GPS data features alone as predictors.

\section{SYSTEM DEVELOPMENT AND DATA COLLECTION}

We developed a smartphone sensing application called $\mathrm{Me}$ for Android using the AWARE framework [7]. Me unobtrusively and passively collects contextual smartphone sensor data shown in Table 1 without interrupting or prompting the user. Me is privacy-aware and does not collect any personal information such as text, voice, telephone numbers, content of applications or web pages visited, except for meta data; for example, the time the screen was locked or unlocked, what application was launched at what a time, and when messages

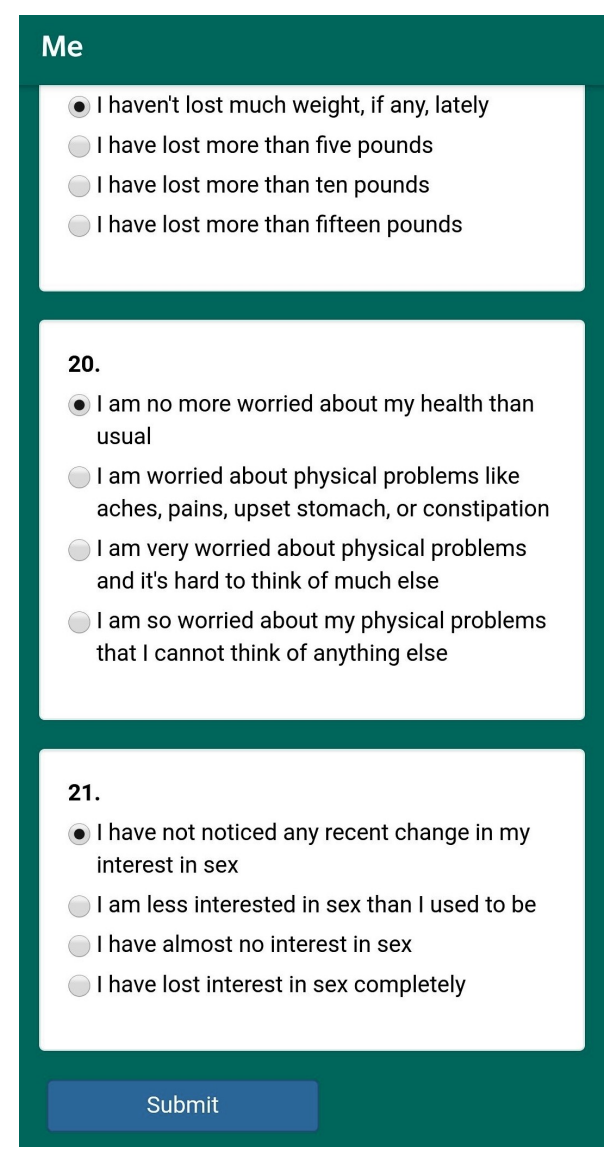

Figure 1: Screenshot of a self report questionnaire on the Me application

were received. The Me application saves the collected sensor data locally on the phone. The data is then transferred on a secured and encrypted connection to a secure MySQL database hosted in the cloud.

In addition to the sensor data, the Me app schedules PHQ9, BDI, and BIG-5 questionnaires based on the frequencies defined in Table 1. When the questionnaire is due, Me shows a notification as seen in Figure 2. The user then taps the notification to open the questionnaire as seen in Figure 1. After providing answers to the questionnaire, the user clicks on the Submit button to save the answers provided. As a remedial measure, Me also shows the questionnaire within the application's main menu, hence the user could access the questionnaire in case they miss the notification. Me does not implement any feedback or data visualisation mechanism at this stage, since presenting such feedback to participants, may cause a change in the behavior we are studying. 
Table 1: Data collected with the Me application

\begin{tabular}{lll}
\hline Sensor Data & Description & Frequency \\
\hline GPS Location & Latitude, Longitude coordinates & \\
$\begin{array}{l}\text { Physical Activity } \\
\text { Light }\end{array}$ & Walking, Running, biking, in Vehicle & Every 5 minutes \\
Noise & $\begin{array}{l}\text { Intensity of ambient light } \\
\text { Intensity of ambient noise }\end{array}$ & \\
\hline Screen Interaction & Screen locks, unlocks, touch, scroll up and down & \\
Battery & Battery level changes, battery charges and discharges & \\
Application & Time, name of applications that are launched & Continuous monitoring \\
Notifications & Time, application name of the notification \\
Calls & Time of call, call type (incoming, Outgoing, missed) & \\
Messages & Time of SMS message, message type (received, sent) & \\
\hline \hline Self Reports & Description & Frequency \\
\hline BIG-5[13] & 50 item personality trait questionnaire & Beginning of the study \\
\hline Mood & A mood rating questionnaire & Morning, afternoon, evening \\
\hline Sleep & Start and end date and time of sleep session & Morning \\
\hline PHQ-9[15] & 9 item depression test questionnaire & Beginning of the study and every two weeks \\
\hline BDI [1] & 21 item depression test questionnaire & Once at onset and every week \\
\hline
\end{tabular}

now

\section{Me has a Mood survey for you \\ Press here to answer the survey}

Figure 2: Notification for scheduled self report questionnaire on the Me application

\section{CONCLUSION AND FUTURE WORK}

Next, we have planned to run a longitudinal study for 3 months with 120 participants (40 not depressed, 40 depressed and 40 high-risk). In this study, the Me application will be used to collect the data described in Table 1. At the end of the study, participants will be invited to a post study debriefing session where they will be asked to reflect and comment on statistics and visualizations of their own data, with a semistructured interview. This reflection will provide valuable information from participants to be used in our analysis, for example to annotate days where the understanding of the data is unclear.

With this study, we plan to collect a wide range of different contextual smartphone sensor data as possible since our analysis methods are post-hoc - we do not concretely know which collected variables will help to produce useful metrics in our analysis. Our approach to the analysis of the data is to create an anomaly detection[11] and entropy analysis methods for monitoring the early onset and progression of MDD. Anomaly detection methods find non conforming patterns to expected behavior in data, is applicable to unlabelled or non-annotated data, taking into consideration the internal structure of the data. Entropy analysis [2, 3, 10] measures the degree of complexity , uncertainty, and disorder of an observed behavior with the passage of time. Instead of PA, our methods will be better suited to detect unexpected growth of anomalous behavior and the deterioration of behaviors such as daily routines, activities, actions, social interactions, over time thus detecting the early onset and progression of MDD. For instance, scenarios that might trigger the detection of anomalous behaviour are;

- Texting in extraordinary hours: unusual phone interaction at extraordinary hours of the day, inferred from contextual sensor data such as time of day, application launches, screen lock and unlock, touch interactions and number of SMS messages.

- Physical and social isolation: disappearance of physical activity, reduced exercising, and social interaction, calling and texting inferred from contextual sensor data such as GPS locations, Physical activity, noise intensity and light intensity. 
- Sleep changes: unusual changes in sleep time, wake up time, and sleep duration inferred from GPS location, physical activity, screen lock and unlock, touch interactions, noise intensity and light intensity

In conclusion, we presented our preliminary work and future directions on developing a system aimed at detecting MDD at the early stages. With our developed Android smartphone application, We plan a longitudinal study to collect a wide range of dataset to build anomaly detection and entropy analysis methods to ultimately develop new metrics for detecting the early onset and progression of depression.

\section{ACKNOWLEDGMENTS}

This work is partially funded by the Academy of Finland (Grants 313224-STOP, 316253,320089-SENSATE and 3189276Genesis Flagship) and Infotech Oulu

\section{REFERENCES}

[1] Aaron T Beck, Calvin H Ward, Mock Mendelson, Jeremiah Mock, and John Erbaugh. 1961. An inventory for measuring depression. Archives of general psychiatry 4, 6 (1961), 561-571. https://doi.org/10.1001/ archpsyc.1961.01710120031004

[2] Przemysław Bereziński, Bartosz Jasiul, and Marcin Szpyrka. 2015. An entropy-based network anomaly detection method. Entropy 17, 4 (2015), 2367-2408. https://doi.org/10.3390/e17042367

[3] Madalena Costa, Chung-Kang Peng, Ary L. Goldberger, and Jeffrey M. Hausdorff. 2003. Multiscale entropy analysis of human gait dynamics. Physica A: Statistical Mechanics and its Applications 330, 1 (2003), 53 60. https://doi.org/10.1016/j.physa.2003.08.022

[4] Thomas H. Davenport. 2014. A Predictive Analytics Primer. Harvard Business Review (Sept. 2014). https://hbr.org/2014/09/a-predictiveanalytics-primer

[5] Ezgi Dogan, Christian Sander, Xenija Wagner, Ulrich Hegerl, and Elisabeth Kohls. 2017. Smartphone-based monitoring of objective and subjective data in affective disorders: where are we and where are we going? Systematic review. Journal of medical Internet research 19, 7 (2017), e262. https://doi.org/10.2196/jmir.7006

[6] Asma Ahmad Farhan, Chaoqun Yue, Reynaldo Morillo, Shweta Ware, Jin Lu, Jinbo Bi, Jayesh Kamath, Alexander Russell, Athanasios Bamis, and Bing Wang. 2016. Behavior vs. introspection: Refining prediction of clinical depression via smartphone sensing data. In 2016 IEEE Wireless Health (WH). IEEE, 1-8. https://doi.org/10.1109/WH.2016.7764553

[7] Denzil Ferreira, Vassilis Kostakos, and Anind K. Dey. 2015. AWARE: Mobile Context Instrumentation Framework. Frontiers in ICT 2 (2015), 6. https://doi.org/10.3389/fict.2015.00006

[8] Eiko I Fried and Randolph M Nesse. 2015. Depression is not a consistent syndrome: an investigation of unique symptom patterns in the STAR* D study. Fournal of affective disorders 172 (2015), 96-102. https: //doi.org/10.1016/j.jad.2014.10.010

[9] Eiko I. Fried and Randolph M. Nesse. 2015. Depression sum-scores don't add up: why analyzing specific depression symptoms is essential BMC Medicine 13, 1 (06 Apr 2015), 72. https://doi.org/10.1186/s12916 015-0325-4

[10] Fariba Ghaffari and Mahdi Abadi. 2015. DroidMalHunter: A novel entropy-based anomaly detection system to detect malicious Android applications. In 2015 5th International Conference on Computer and Knowledge Engineering (ICCKE). IEEE, 301-306. https://doi.org/10. 1109/ICCKE.2015.7365846
[11] Markus Goldstein and Seiichi Uchida. 2016. A Comparative Evaluation of Unsupervised Anomaly Detection Algorithms for Multivariate Data. PLOS ONE 11 (04 2016), 1-31. https://doi.org/10.1371/journal.pone. 0152173

[12] Ann M Hemmerle, James P Herman, and Kim B Seroogy. 2012. Stress, depression and Parkinson's disease. Experimental neurology 233, 1 (2012), 79-86. https://doi.org/10.1016/j.expneurol.2011.09.035

[13] John A. Johnson. [n. d.]. Administering IPIP Measures, with a 50item Sample Questionnaire. https://ipip.ori.org/New_IPIP-50-itemscale.htm

[14] Ronald C. Kessler. 2012. The Costs of Depression. The Psychiatric Clinics of North America 35, 1 (March 2012), 1-14. https://doi.org/10. 1016/j.psc.2011.11.005

[15] Kurt Kroenke, Robert L Spitzer, and Janet BW Williams. 2001. The PHQ9: validity of a brief depression severity measure. Fournal of general internal medicine 16, 9 (2001), 606-613. https://doi.org/10.1046/j.15251497.2001.016009606.x

[16] World Health Organisation. 2018. Depression. https://www.who.int/ news-room/fact-sheets/detail/depression

[17] Georgiy S Pushkarev, Vadim A Kuznetsov, Yakov A Fisher, Anna M Soldatova, and Tatiana N Enina. 2018. Depression and all-cause mortality in patients with congestive heart failure and an implanted cardiac device. Turk Kardiyoloji Dernegi arsivi: Turk Kardiyoloji Derneginin yayin organidir 46, 6 (2018), 479-487. https://doi.org/10.5543/tkda. 2018.04134

[18] Darius A Rohani, Maria Faurholt-Jepsen, Lars Vedel Kessing, and Jakob E Bardram. 2018. Correlations between objective behavioral features collected from mobile and wearable devices and depressive mood symptoms in patients with affective disorders: Systematic review. FMIR mHealth and uHealth 6, 8 (2018), e165. https: //doi.org/10.2196/mhealth.9691

[19] Rui Wang, Weichen Wang, Alex daSilva, Jeremy F Huckins, William M Kelley, Todd F Heatherton, and Andrew T Campbell. 2018. Tracking depression dynamics in college students using mobile phone and wearable sensing. Proceedings of the ACM on Interactive, Mobile, Wearable and Ubiquitous Technologies 2, 1 (2018), 43. https: //doi.org/10.1145/3191775 